\title{
Une géographie des mouvements de jeunesse en Belgique francophone
}

A geography of youth movements in French-speaking Belgium

\section{Dominique Dubruille, Benjamin Wayens et Jean-Michel Decroly}

\section{(2) OpenEdition}

\section{Journals}

\section{Édition électronique}

URL : http://journals.openedition.org/belgeo/12295

DOI : $10.4000 /$ belgeo.12295

ISSN : 2294-9135

Éditeur :

National Committee of Geography of Belgium, Société Royale Belge de Géographie

\section{Édition imprimée}

Date de publication : 30 septembre 2005

Pagination : $307-326$

ISSN : 1377-2368

\section{Référence électronique}

Dominique Dubruille, Benjamin Wayens et Jean-Michel Decroly, « Une géographie des mouvements de jeunesse en Belgique francophone », Belgeo [En ligne], 3 | 2005, mis en ligne le 28 octobre 2013, consulté le 05 février 2021. URL : http://journals.openedition.org/belgeo/12295; DOI : https://doi.org/ 10.4000/belgeo.12295

Ce document a été généré automatiquement le 5 février 2021.

Belgeo est mis à disposition selon les termes de la licence Creative Commons Attribution 4.0 International. 


\title{
Une géographie des mouvements de jeunesse en Belgique francophone
}

\author{
A geography of youth movements in French-speaking Belgium \\ Dominique Dubruille, Benjamin Wayens et Jean-Michel Decroly
}

\section{Introduction}

1 Réelle innovation pédagogique, l'apparition de groupements structurés de jeunes dans un but de divertissement a apporté une nouvelle donne au paysage socio-culturel belge au début du XXe siècle. Les mouvements de jeunesse sont tellement intégrés dans notre société, que nous avons l'impression qu'ils ont toujours existé, et ce partout en Belgique. Pourtant, le fait est que cette ubiquité n'est pas une réalité, particulièrement si chacun des mouvements est envisagé individuellement. Dès lors, on peut se demander dans quelle mesure la géographie des mouvements de jeunesse traduit leur histoire et leurs liens avec l'évolution générale de la société.

2 C'est à ces questions que nous allons tenter de répondre, sur base d'éléments, statistiques, bibliographiques ou oraux, pour expliciter la géographie des mouvements de jeunesse en Belgique francophone. L'approche géographique apporte une dimension inédite à l'analyse de ce phénomène. Elle donnera une meilleure vision de l'encadrement socio-culturel offert par ces mouvements, contribuant ainsi modestement à une géographie de la société civile (voir pour une vision élargie, Vandermotten et al., 2005).

3 Le nombre de jeunes dans les mouvements de jeunesse en Communauté française approche les 100000 . Sur 100 jeunes de 5 à 24 ans, habitant en Communauté française, 8,6 d'entre eux sont membres d'un mouvement de jeunesse. Cette proportion est assez élevée quand on sait que plus de $50 \%$ des membres ont entre 8 et 15 ans. Il y a donc un réel intérêt à analyser ce phénomène de plus près. A titre de comparaison, les mouvements flamands regroupent près de 220000 affiliés, ce qui donne en taux d'encadrement des 5-20 ans autour de $13 \%$. Cette différence est cohérente lorsque l'on 
connaît l'importance de l'encadrement catholique en Flandre, notamment au travers de l'enseignement, qui est un espace privilégié de recrutement du scoutisme.

4 L'hégémonie catholique est telle que l'analyse spatiale des mouvements de jeunesse, tant en Belgique francophone qu'en Flandre, passe par une distinction entre les différents mouvements de l'obédience catholique. Ce qui fait la spécificité de la partie francophone du pays, c'est une présence plus importante, bien que toujours relativement modeste, des mouvements non chrétiens ( $8,2 \%$ des affiliés chez les Scouts et Guides Pluralistes et Faucons Rouges contre seulement 6,9 pour les Federatie voor Open Scoutisme, pluralistes, en Flandre) et la non unification des Scouts et Guides francophones (réunis en Vlaams Verbond van Katholieke Scouts en Meisjesgidsen en Flandre).

Figure 1. Structure des affiliés par mouvements, Flandre et Belgique francophone.

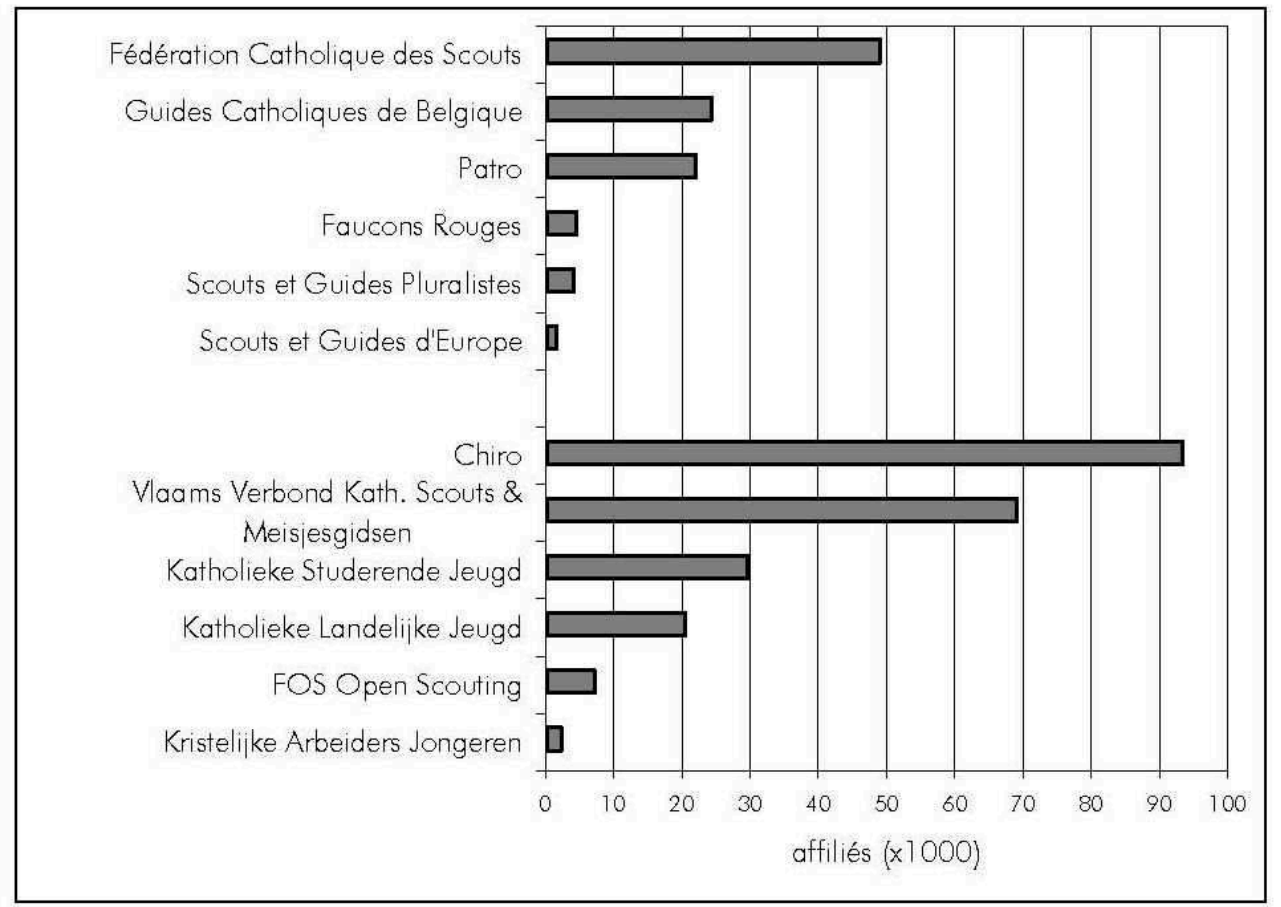

Source : données d'affiliation des fédérations.

\section{Mouvement de jeunesse : définition et données disponibles}

5 Un mouvement de jeunesse est une organisation, constituée de plusieurs groupements locaux, ayant pour objet, dans le cadre d'un projet qui lui est propre, l'éducation, l'animation et l'occupation d'enfants ou de jeunes au travers d'un ensemble d'activités variées.

6 Les mouvements de jeunesse retenus dans la présente analyse conjuguent deux caractéristiques. Primo, ils figurent sur la liste officielle, établie par la Communauté française de Belgique, des associations reconnues en tant que mouvements de jeunesse. Secundo, ils remplissent simultanément des critères d'ordre économique (organisation 
sans but lucratif), fonctionnel (réunions organisées à intervalle régulier) et thématique (activités diversifiées): il s'agit d'associations bénévoles, dont les réunions sont hebdomadaires et qui proposent des activités axées sur le jeu, la rencontre avec la nature et les contacts humains. Ne sont donc pas prises en compte les associations telles que les clubs sportifs, maisons de jeunes, ... qui ne répondent pas à la définition habituelle d'un mouvement de jeunesse.

7 Au total, six mouvements ont été identifiés : la Fédération Nationale des Patros filles et garçons (F.N.P.F. et F.N.P.), la Fédération Catholique des Scouts Baden-Powell (F.C.S. BP), les Guides Catholiques de Belgique (G.C.B.), les Scouts et Guides Pluralistes (S.G.P.), les Faucons Rouges et les Guides et Scouts d'Europe. Le dernier n'a toutefois pas été inclus dans l'analyse car, en raison de son apparition récente, il rassemble trop peu de groupements locaux.

8 Les données relatives aux cinq organisations retenues sont de trois types: études écrites, témoignages oraux et statistiques. Les premières, peu nombreuses (quelques articles dans des revues spécialisées) ou difficiles d'accès (mémoires universitaires), sont essentiellement historiques. Elles portent pour la plupart sur un mouvement particulier et/ou sur la période antérieure à la Deuxième Guerre mondiale. Il existe en revanche de nombreux témoins de l'évolution des mouvements depuis la période charnière des années 1970. Cette période de transition marquera un tournant décisif en ce qui concerne la pédagogie de l'enfant. De nouvelles méthodologies et projets éducatifs voient ainsi le jour dans l'ensemble du secteur de la jeunesse. Nous en avons rencontré et interrogé une vingtaine, issus des cinq mouvements analysés, qui occupèrent ou occupent encore des fonctions importantes au faite de la hiérarchie de leur fédération et jouèrent un rôle actif dans la promotion de celle-ci. Les entretiens furent réalisés selon un canevas pré-établi de questions ouvertes portant sur l'histoire de chaque mouvement, les personnes interrogées étant amenées dans ce cadre à commenter les cartes de distribution spatiale des affiliés préalablement réalisées.

9 Les données statistiques, enfin, proviennent des listes d'affiliation 2001-2002 des fédérations, complétées, pour la Fédération Catholique des Scouts et les Guides Catholiques de Belgique, par des informations sur la répartition des membres selon l'âge et le sexe.

\section{Géographie de l'encadrement par le scoutisme}

La figure 2 donne la répartition des affiliés au lieu de réunion, toutes fédérations confondues. Les principales concentrations s'observent à Bruxelles, dans le Brabant wallon, ainsi que tout au long du sillon wallon. Dans les zones moins densément peuplées la présence des mouvements se caractérise essentiellement par une implantation dans les petites villes. 
Figure 2. A (haut) : Nombre d'affiliés à une fédération relevant du scoutisme (y compris les Scouts d'Europe). Les affiliés sont cartographiés dans la commune où est localisé le siège principal des activités. $B$ (bas) : Part des 5-24 affiliés à une fédération. Les membres étant enregistrés au siège des activités et la population des 5-24 ans au domicile, ce taux néglige toutefois l'influence d'un mouvement hors de sa commune d'ancrage.

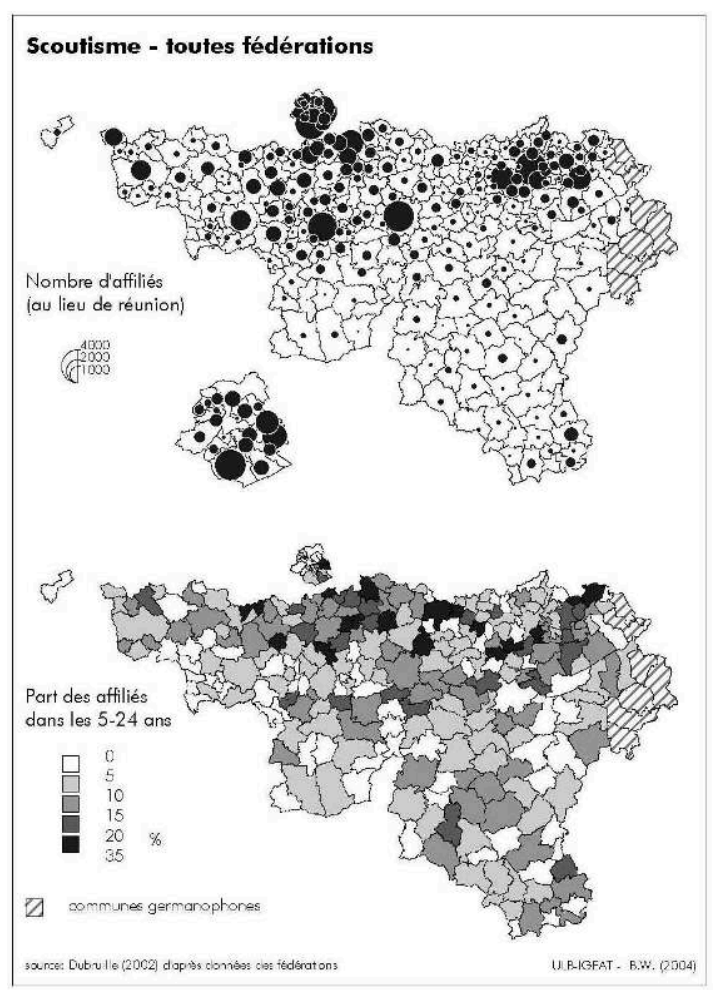

Sources : Dubruille (2002) d'après données des fédérations ; INS

11 Cette géographie, très urbaine de prime abord, doit cependant être fortement nuancée. C'est l'objet de la carte qui relativise les effectifs en représentant la proportion de jeunes de 5-24 ans fréquentant un mouvement de jeunesse. Elle montre clairement que les plus fortes fréquentations relatives ne se rencontrent pas au coeur des régions urbaines mais dans les banlieues. Cette répartition spatiale est également clairement lisible dans le tableau 1 qui fournit la proportion d'affiliés selon le degré d'urbanisation des communes. Tant dans la sphère bruxelloise (Bruxelles et Brabant wallon) que dans le reste de la Belgique francophone, les plus forts taux de pénétration se rencontrent en banlieue. Les mouvements de jeunesse s'y sont massivement implantés à partir des années 1970, dans le cadre d'une périurbanisation résidentielle qui a impliqué les groupes sociaux les plus pourvoyeurs de membres et d'animateurs pour ces associations.

Evoquons également une présence non négligeable de certaines fédérations francophones en Flandre, dont la majorité des effectifs relève de la Fédération des Guides Catholiques. Ces unités sont essentiellement présentes dans la banlieue Bruxelloise (Brabant flamand), dans la région d'Anvers et accessoirement à Gand. Pour ces deux dernières localisations, cet héritage historique date d'avant la communautarisation des fédérations qui s'opéra entre 1929 et 1966. Ces implantations sont indéniablement liées à la présence, à l'époque, d'une bourgeoisie flamande francophone. 
13 Du fait de la structuration sociale des agglomérations urbaines, la différentiation selon le degré d'urbanisation se double d'une différentiation selon des critères socioéconomiques. Ne disposant pas du lieu de résidence des membres, il est difficile d'établir un vrai profil socio-économique. On peut toutefois l'approcher en analysant la distribution spatiale selon une estimation du niveau de vie de la commune où sont installées les unités. Trois groupes de communes (faible, moyen, et élevé) ont été définis en fonction du revenu fiscal moyen par déclaration et les taux de pénétration calculés dans chacun d'entre eux. Tous mouvements confondus, 13,5 \% des 5-24 ans des communes à revenu fiscal élevé fréquentent un mouvement de jeunesse contre 7,9\% pour des communes à revenu fiscal moyen et $4,2 \%$ pour les communes à revenu fiscal faible. Un gradient selon le niveau de revenu apparaît donc clairement. Bruxelles en est un très bon exemple : alors que les 19 communes bruxelloises sont toutes urbaines dans la première typologie, on observe les plus forts taux de pénétration dans les communes du cadran sud-est, le plus aisé de l'agglomération. Dans certaines d'entre elles, les membres de mouvements de jeunesse représentent près de $30 \%$ des jeunes de 5-24 ans.

\section{Cinq mouvements, trois géographies?}

14 La géographie actuelle des mouvements de jeunesse est le produit de leur diffusion spatiale et des facteurs qui la surdéterminèrent. Au cours de la première moitié du XX siècle, les mouvements étudiés se sont implantés dans les régions les plus peuplées, là où il existait une demande pour ce type de loisirs et où il était facile de rassembler le minimum de participants pour le bon fonctionnement d'un mouvement. La jeunesse de l'époque n'avait en réalité que peu de loisirs à sa disposition, et dans un contexte de prolongation de la scolarisation, les villes assistèrent les premières au développement du scoutisme. Quant aux zones rurales, elles échappèrent largement au processus de cette première moitié $\mathrm{du} \mathrm{XX}^{\mathrm{e}}$ siècle, car la jeunesse n'y était pas très réceptive et pas assez concentrée spatialement.

Les différents mouvements ont ensuite connu, selon des modalités variables, une vague d'expansion spatiale, allant des zones peuplées vers les régions les plus reculées et les moins habitées. Pour comprendre ces processus contrastés de diffusion, il importe de se pencher sur l'histoire de chaque mouvement. Dans le cadre de cette démarche historique, une attention particulière sera accordée aux relations entretenues entre les mouvements et les trois piliers qui régissent la société belge, structurant depuis longtemps une bonne partie du monde associatif, à savoir les piliers chrétien, socialiste et libéral.

16 En effet, ces relations expliquent en partie le profil sociologique de la population touchée par les mouvements de jeunesse ainsi que leur diffusion spatiale. Comment ne pas constater que les espaces où les mouvements de jeunesse, essentiellement catholiques, encadrent une part importante des jeunes sont aussi les espaces où l'enseignement catholique domine le paysage scolaire? Comment ne pas associer la répartition actuelle des Faucons Rouges aux terres d'hégémonie socialiste et celle des Scouts et Guides Pluralistes au foyer du libéralisme belge que constitue la Région bruxelloise?

17 Pour mener à bien l'analyse de la répartition géographique des différents mouvements, nous croiserons systématiquement la localisation actuelle des unités (figures 3, 4 et 5) avec le background historique propre à chaque obédience. Le Tableau 2 offre une 
visualisation globale et comparative de cet historique pour les cinq mouvements de jeunesse. Les principaux facteurs de développement et de diffusion spatiale y sont abordés au travers de trois époques, caractérisées chacune par une vision de la pédagogie de l'enfant, des buts recherchés à travers le scoutisme et des volontés d'appartenance à un pilier spécifique.

Figure 3. Répartition géographique des affiliés à la Fédération Catholique des Scouts et aux Guides Catholiques de Belgique.

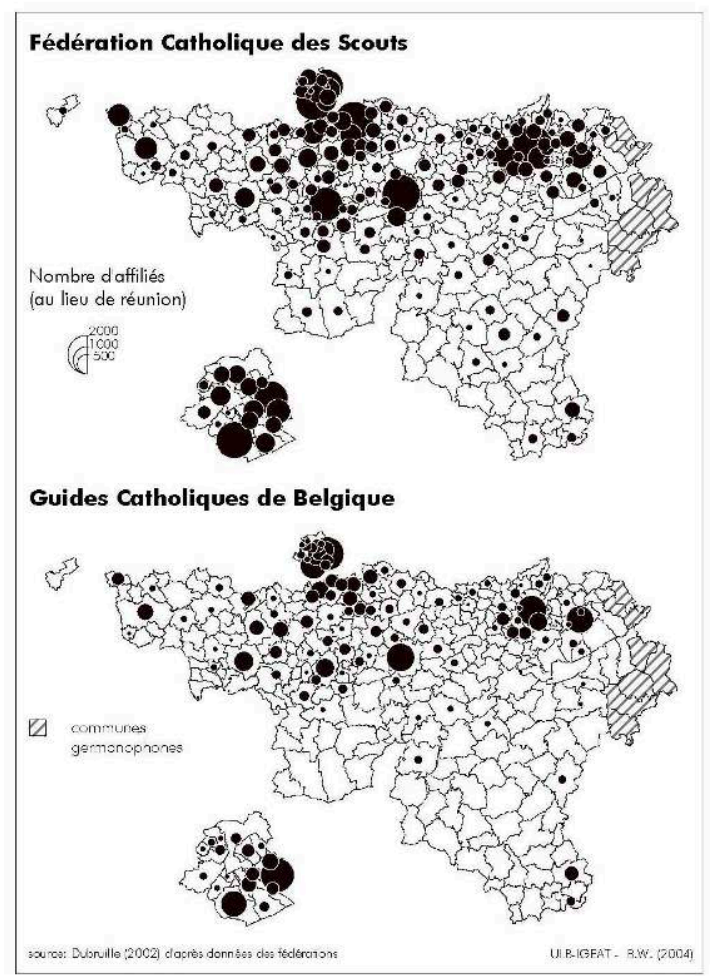

Source : Dubruille (2002), d'après données des fédérations. 
Figure 4. Répartition géographique des affiliés du Patro.

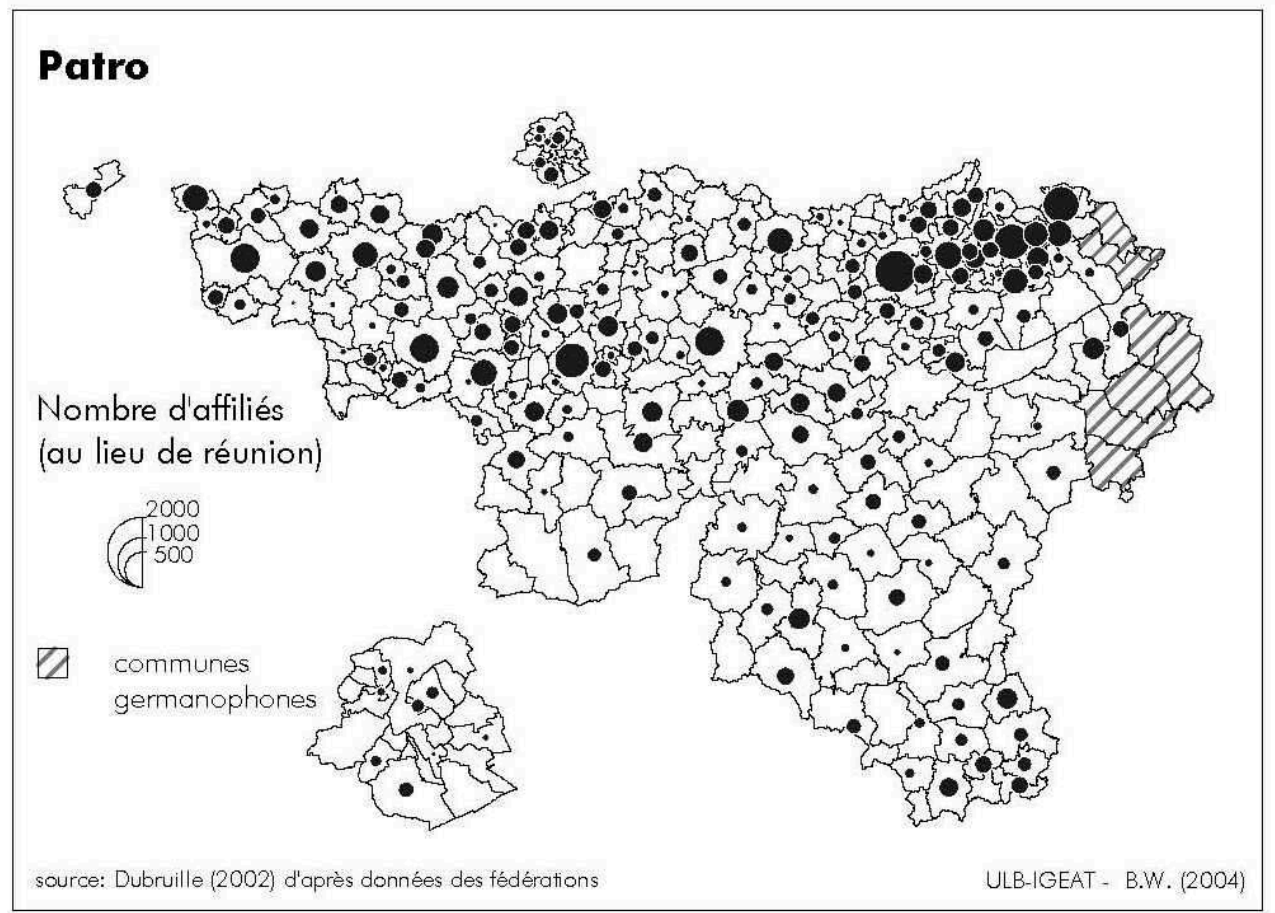

Source : Dubruille (2002), d'après données des fédérations.

Figure 5. Répartition géographique des affiliés aux Faucons Rouges et Scouts et Guides Pluralistes.

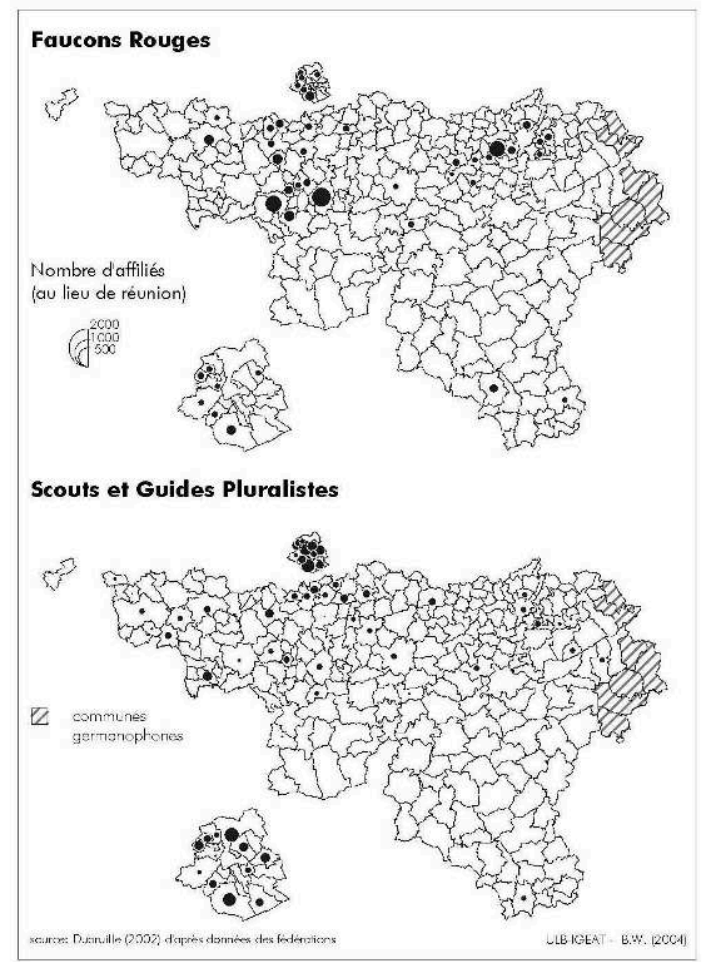

Source : Dubruille (2002), d'après données des fédérations. 
Tableau 2. Synthèse de l'évolution des caractéristiques des différents mouvements.

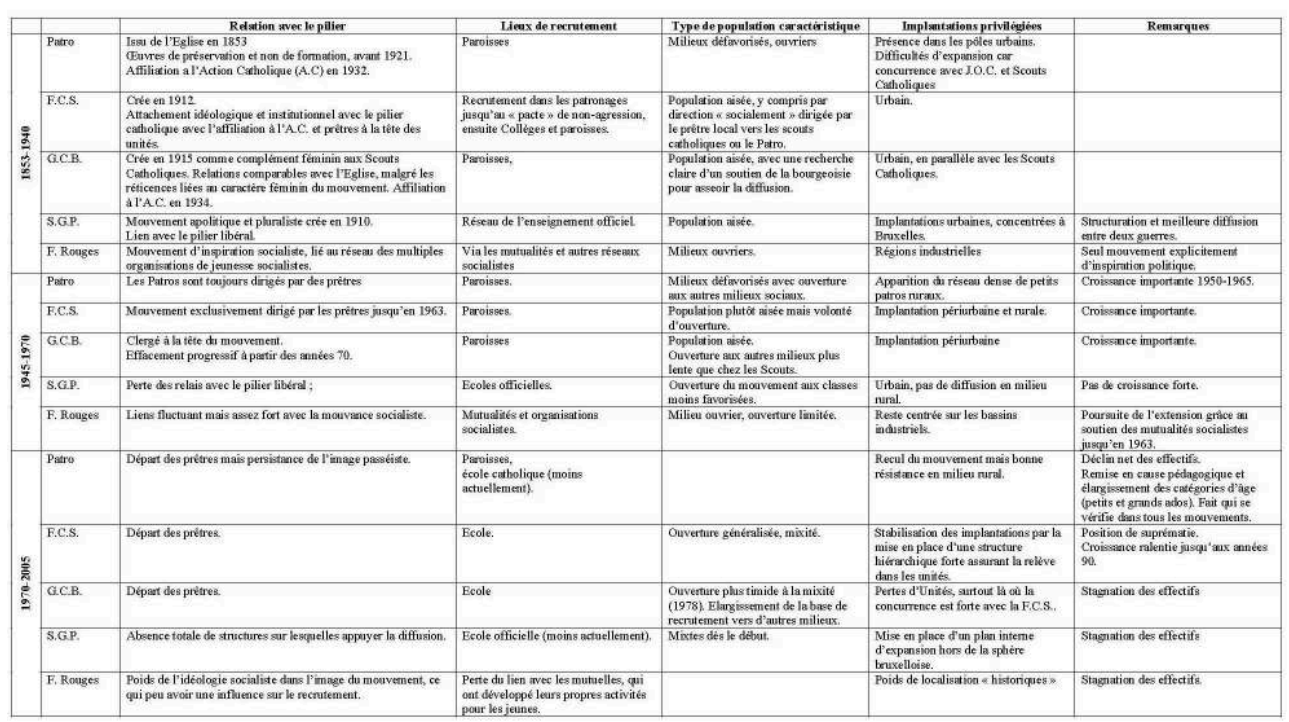

\section{Scouts et guides catholiques : une géographie urbaine}

\section{La fédération catholique des scouts}

Scouts et guides catholiques ont des répartitions spatiales semblables, privilégiant nettement l'urbain et la banlieue dans l'ensemble de la Belgique francophone. Les deux fédérations sont particulièrement bien ancrées dans la sphère bruxelloise. Alors que le poids catholique est historiquement faible dans l'électorat bruxellois (Vandermotten et al., 2001), plus de $50 \%$ des membres de mouvements de jeunesse présents dans la capitale font partie de la Fédération Catholique des Scouts (F.C.S.).

Cette situation trouve son origine dans l'implantation précoce des scouts catholiques à Bruxelles : non seulement, les unités bruxelloises de la F.C.S. furent parmi les premières à être créées par le mouvement scout catholique mais encore elles restèrent pendant longtemps les principales à opérer à Bruxelles grâce à leur recrutement privilégié dans les grandes écoles de l'enseignement libre. Pour y maintenir sa position privilégiée, la F.C.S. a pratiqué une politique volontariste d'expansion spatiale, notamment au cours des années 1960, dans le cadre de la croissance généralisée des mouvements de jeunesse. Il en résulte que les scouts F.C.S. sont à l'heure actuelle les seuls à couvrir la quasi totalité des communes bruxelloises (à l'exception de St. Josse). La fédération s'est ensuite implantée en Brabant wallon, dans le cadre de périurbanisation des mouvements de jeunesse présents à Bruxelles. Elle y a rencontré un franc succès, car elle s'adressait pour l'essentiel à un segment de population issue de la capitale, où les scouts catholiques occupaient déjà une position privilégiée.

Dans le Namurois aussi, la majorité des membres d'unités urbaines font partie des scouts et guides catholiques. A Liège, la présence des deux fédérations est également très forte. On observera toutefois ici, tout comme dans le reste de la Wallonie, une pénétration plus forte de guides en ville et dans la proche banlieue, ce qui les différentie des scouts catholiques. Dans le Hainaut, enfin, les différentes unités se situent plutôt en périphérie et sont quasiment absentes des communes les moins 
favorisées, ayant des pourcentages élevés d'étrangers, de chômage et de personnes faiblement qualifiées.

21 Aujourd'hui, la Fédération Catholique des Scouts B-P occupe la place dominante dans le paysage des mouvements de jeunesse, avec en 2001, 45845 membres et 428 unités. La forte spécificité urbaine et bourgeoise du mouvement apparaîtra très rapidement dans son histoire. Bien qu'à l'origine le scoutisme ait été pensé pour pallier le manque d'activités proposées aux jeunes des banlieues ouvrières, en Belgique, on observe toutefois très rapidement que le mouvement des scouts catholiques s'adresse essentiellement à la petite bourgeoisie. Et cette dernière est évidemment plutôt concentrée dans les pôles urbains. Ceci explique une présence d'unités " historiques » dans cet environnement.

Dès 1912, date d'apparition de la première unité, le mouvement véhicula une idéologie forte de patriotisme, d'entraide et de fraternité, où la hiérarchie occupait une place importante. Dans ses fondements, le scoutisme de Baden-Powel ne revendique pas de confession, mais dans un premier temps, le mouvement des scouts catholiques fit de la religion une priorité.

A propos des relations entre l'Eglise et les mouvements de jeunesse, il est important de bien faire la différence entre le Patro, d'une part, les Scouts et guides catholiques, d'autre part. Dès l'origine, les scouts et guides catholiques, contrairement au Patronage, n'ont pas été des mouvements dépendants de l'Eglise, mais plutôt des mouvements en connexion avec l'Eglise. Si, dans un premier temps, le clergé a eu une place omniprésente à tous les échelons de la hiérarchie, très vite les fédérations ont exprimé leur désaccord face à ce qu'ils percevaient comme une récupération du mouvement par l'Eglise. En dépit de ces réticences, les prêtres gardèrent la mainmise sur les scouts et guides catholiques jusqu'en $1963^{1}$. A cette date, il ne fût plus nécessaire d'obtenir l'autorisation des curés pour ouvrir de nouvelles implantations de troupes ou d'unités dans une paroisse.

De par leurs rapports un peu moins intimes avec l'Eglise, les scouts et guides catholiques ont sans doute moins bénéficié des structures paroissiales que les patros. Les troupes scoutes non paroissiales, comme les troupes des Collèges, restèrent toutefois nettement minoritaires jusqu'en 1970, représentant, selon François Rykmans (cadre fédéral et adjoint de la Fédération des Scouts Catholiques entre 1975 et 1980), à peine $10 \%$ des unités. Elles ne prirent leur réel essor qu'à la suite de la rupture de 1963.

\section{Les guides catholiques}

25 Aujourd'hui, la G.C.B. s'impose en tant que deuxième mouvement d'importance numérique, avec 249 unités et 23331 affiliés en 2002, dont plus de 2000 membres ne se réunissent pas sur le territoire de la Communauté française.

La présence des guides catholiques est dans la grande majorité des cas corrélée avec celle des scouts catholiques. Comme ces derniers, les G.C.B. sont particulièrement bien présents dans les communes aux revenus fiscaux élevés, ainsi que dans les zones plus urbanisées (tableau 1). Le faible poids relatif des guides en Brabant wallon par rapport aux scouts catholiques, alors que les deux mouvements sont arrivés à la même époque, s'explique largement, par une ouverture plus tardive des guides à la coéducation (mixité). La proportion de garçons dans les unités G.C.B. de cette région ne dépasse pas la moyenne générale, à savoir $8 \%$ d'effectifs masculins. 
Tableau 1. Part des 5-24 affiliés par fédération selon l'urbanisation et le revenu moyen des communes où est localisé le siège principal des activités.

\begin{tabular}{|c|c|c|c|c|c|c|c|c|}
\hline \multicolumn{3}{|c|}{$\begin{array}{c}\% \text { des affiliés dans la population } \\
\text { des } 5-24 \text { ans }\end{array}$} & $\begin{array}{l}\text { Tous } \\
\text { mouvements }\end{array}$ & $\begin{array}{l}\text { Fédération } \\
\text { Catholique } \\
\text { des Scouts }\end{array}$ & $\begin{array}{c}\text { Guides } \\
\text { Catholiques } \\
\text { de Belgique }\end{array}$ & $\begin{array}{c}\text { Scouts et } \\
\text { Guides } \\
\text { Pluralistes }\end{array}$ & Patro & $\begin{array}{l}\text { Faucons } \\
\text { Rouges }\end{array}$ \\
\hline \multirow{6}{*}{ 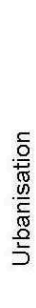 } & \multicolumn{2}{|c|}{ Wallonie + Bruxelles } & $8,6 \%$ & $4,0 \%$ & $1,9 \%$ & $0,3 \%$ & $1,9 \%$ & $0,4 \%$ \\
\hline & Sphère & ville & $6,8 \%$ & $3,5 \%$ & $2,0 \%$ & $0,6 \%$ & $0,2 \%$ & $0,2 \%$ \\
\hline & Bruxelloise & banlieue & $13,2 \%$ & $7,3 \%$ & $3,5 \%$ & $0,6 \%$ & $1,4 \%$ & $0,4 \%$ \\
\hline & & ville & $8,0 \%$ & $3,9 \%$ & $2,3 \%$ & $0,2 \%$ & $1,2 \%$ & $0,4 \%$ \\
\hline & Wallonie & banlieue & $9,0 \%$ & $4,4 \%$ & $1,6 \%$ & $0,2 \%$ & $2,4 \%$ & $0,4 \%$ \\
\hline & & rural & $8,7 \%$ & $3,3 \%$ & $1,4 \%$ & $0,2 \%$ & $3,3 \%$ & $0,4 \%$ \\
\hline \multirow{3}{*}{ 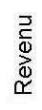 } & \multirow{3}{*}{$\begin{array}{c}\text { revenu fiscal } \\
\text { moyen de la } \\
\text { commune }\end{array}$} & faible $(25 \%)$ & $4,2 \%$ & $1,8 \%$ & $0,7 \%$ & $0,2 \%$ & $1,2 \%$ & $0,3 \%$ \\
\hline & & moyen (50\%) & $7,9 \%$ & $3,3 \%$ & $1,6 \%$ & $0,3 \%$ & $2,2 \%$ & $0,4 \%$ \\
\hline & & élevé (25\%) & $13,5 \%$ & $7,1 \%$ & $3,5 \%$ & $0,5 \%$ & $1,9 \%$ & $0,3 \%$ \\
\hline
\end{tabular}

Sont considérées comme «ville » les communes centrales des régions urbaines et les communes de forte urbanisation morphologique et fonctionnelle contiguës. La «banlieue » est constituée par les autres communes des régions urbaines. La classification des communes selon le revenu est établie de façon à ventiler $25 \%$ de la population totale dans la classe « faible revenu », $50 \%$ dans une classe intermédiaire et $25 \%$ dans la classe « haut revenu ».

Sources : Dubruille (2002), d'après données des fédérations ; INS ; Mérenne-Schoumaker B., Van der Haegen H. et al. (1998).

La coïncidence globale des géographies est évidemment à associer au fait que le développement des guides catholiques a été très longtemps lié à celui des scouts catholiques. Apparues en 1915, seulement quelques années après les premières troupes de scouts, les guides se présentèrent d'emblée comme leur pendant féminin. Ce lien étroit sur le plan des objectifs se doubla d'une étroite correspondance géographique entre les deux mouvements: dans bien des cas, l'implantation préalable de scouts catholiques fut à l'origine de l'apparition du mouvement féminin ${ }^{2}$. C'est ainsi qu'aujourd'hui, une grande majorité des implantations G.C.B. se trouvent à proximité de celles de la F.C.S.

Dans un contexte idéologique marqué par une forte présence du catholicisme à tous les échelons de la société et un puritanisme exacerbé, on envisageait difficilement la naissance d'un mouvement de jeunesse en marge de l'Eglise (Stavaux, 1995, p.27), surtout s'il était réservé aux filles. Les guides catholiques se positionnèrent donc d'emblée comme mouvement confessionnel.

29 La cellule de reproduction et d'implantation de base des guides resta longtemps la paroisse : elle fournissait toute la logistique dont le mouvement avait besoin (locaux, recrutement, cadres...). De plus, dans un premier temps, la plupart des demandes de création de nouvelles unités émanaient directement des autorités ecclésiastiques locales, sans répondre à un plan d'expansion bien établi. Par ce biais, le pilier catholique souhaitait clairement "drainer» les jeunes filles vers des mouvements chrétiens, afin de poursuivre le contrôle qu'ils opéraient depuis longtemps via le catéchisme, les patronages...

30 L'implantation initiale des guides en Belgique se heurta à plusieurs obstacles, en particulier le blocage par les prêtres de nombreux projets de compagnies. Afin 
d'obtenir une certaine crédibilité et de contourner l'hostilité du clergé local, les fondateurs du mouvement cherchèrent la reconnaissance de la haute société et des hautes autorités ecclésiastiques. Dans cette optique, ils choisirent, au même titre que les scouts catholiques et le Patronage de s'affilier en 1934 à l'Action Catholique, organisme regroupant les divers mouvements de jeunesse catholiques (Stavaux, 1995). De par leur participation et leur droit de parole en tant que membres de l'Action Catholique, ils purent ainsi contrer plus facilement les prêtres réticents, ce qui favorisa grandement la diffusion du mouvement. L'affiliation à l'Action Catholique permit en outre aux guides de se faire un nom et une place dans le paysage des mouvements de jeunesse en Belgique. Cette nouvelle réputation, contribua à renforcer le caractère bourgeois du milieu social des membres ${ }^{3}$.

31 L'apparition de la mixité dans le scoutisme eut un impact non négligeable sur la fréquentation des troupes de guides. Dans les années 1970-80 de nombreuses unités G.C.B. en difficulté disparurent dans la province du Luxembourg et de Namur, souvent suite à une absorption par les unités locales de scouts catholiques, ouvertes plus précocement à la fréquentation féminine ${ }^{4}$. De la même manière, la vague d'implantation dans les banlieues des scouts catholiques prit plus d'ampleur en se faisant simultanément avec leur ouverture à la mixité. En effet, contrairement à la F.C.S., le mouvement féminin ne proposait pas encore à l'époque des unités mixtes. Dans un contexte de création de nouvelles unités dans les espaces périurbains, de nombreuses familles ayant des garçons et des filles se sont alors tournées vers les scouts, par mesure de facilité. En quelque sorte, l'innovation constituée par la mixité, mieux prise en compte par les scouts, conjuguée au développement de nouvelles unités permis par la croissance démographique, a renforcé leur position relative. Pas étonnant dès lors, que les troupes de scouts catholiques affichent dans le Brabant wallon leurs meilleurs taux de mixité, avec près de $94 \%$ d'unités mixtes, contre une moyenne générale de $78 \%$ pour l'ensemble du mouvement : ce sont les unités les plus récentes.

\section{Patros : une spécificité rurale forte}

Issus de l'offensive hégémonique de l'Eglise catholique du début du $\mathrm{XX}^{\mathrm{e}}$ siècle, et dirigés par des membres du clergé, les patronages (ancienne dénomination du Patro) étaient à l'époque l'un des instruments privilégiés destinés à préserver la jeunesse de la supposée dépravation des moeurs et des courants matérialistes qui gagnaient la société. C'est dans ce contexte qu'ils connurent leur première phase d'expansion.

Le but de cette oeuvre était double. Tout d'abord, il était primordial pour le pilier catholique de préserver les enfants ouvriers des dangers de la rue... et des influences socialistes. Deuxièmement, dans un contexte où la désertion des enfants vis-à-vis de la messe était de plus en plus fréquente, l'Eglise de l'époque voulait approfondir et entretenir les connaissances chrétiennes de ses membres. C'est ainsi que les oeuvres devinrent une sorte de catéchisme après l'école.

L'arrivée du scoutisme en Belgique provoqua une remise en question de l'orientation initiale des patronages. Perçus par une grande partie de la jeunesse comme une institution vieillotte et protectrice dont le paternalisme faisait fuir plus d'un jeune, incapables d'offrir des activités susceptibles de répondre à la demande locale, ils cherchèrent à offrir du neuf. C'est ainsi que l'on vit apparaître les premières unités scoutes au sein même des patronages. Le phénomène resta toutefois limité car le clergé 
voulait éviter la concurrence entre les deux institutions. Par ailleurs, le patronage s'inspira de la méthode du scoutisme dans ses activités, et établit un pacte de non agression avec les scouts catholiques (qui à l'époque étaient également supervisés par l'Eglise) stipulant qu'aucune nouvelle implantation scoute ne serait autorisée là ou se trouvaient des patros ${ }^{5}$. Les prémices d'une ségrégation socio-économique entre patros (implantés d'abord en milieu urbain et ouvrier), Scouts et Guides catholiques furent ainsi mis en place par le clergé présent à la tête des différents mouvements locaux.

Et si, aujourd'hui, le Patro n'a plus grand chose de commun avec ce que furent autrefois les patronages, il a cependant conservé leur héritage historique, à savoir les implantations paroissiales, même si le lien n'est plus systématique. Les valeurs prônées aujourd'hui par le mouvement sont axées sur la solidarité et le développement de l'enfant à travers le groupe, avec comme spécificité un esprit très familial souvent favorisé par la taille restreinte des unités. En 2002, ce mouvement totalisait 21049 membres, dans 373 patros.

Les deux fédérations des patros présentent une répartition plus uniforme que celle des scouts et guides catholiques et une pénétration généralement faible en ville. Cette géographie, en désaccord avec la répartition générale de la population, est la traduction de la spécificité rurale forte de ce mouvement.

Les implantations rurales sont pourtant apparues avec près de 50 ans de retard sur les implantations urbaines. Cette expansion tardive des mouvements de jeunesse en zones rurales s'explique par plusieurs facteurs. Tout d'abord, la demande locale a été plus tardive. Les activités proposées à l'origine par les mouvements de jeunesse étaient principalement axées sur un retour à la nature et avaient donc moins de sens pour les jeunes des campagnes. De plus, le contrôle social plus présent en milieu rural rendait moins urgente la mission moralisatrice du Patronage. Par ailleurs, le travail des jeunes dans les exploitations familiales était encore très répandu, ce qui ne leur laissait pas beaucoup de temps pour fréquenter un mouvement de jeunesse. Et quand on se penche d'un peu plus près sur les mouvements présents en régions rurales, au début du $\mathrm{XX}^{\mathrm{e}}$ siècle, on remarque que dans la plupart des cas, il s'agissait d'une présence unique : la Jeunesse Ouvrière Chrétienne (J.O.C.). Celle-ci a pendant longtemps concurrencé les Patronages.

L'apparition des patros en milieu rural ne s'est donc faite que progressivement, avec d'abord des implantations dans les localités importantes, puis dans de plus petits villages. Ils mirent du temps à atteindre les localités les plus retirées. Bien qu'aujourd'hui le Patro soit encore le mouvement le mieux implanté dans ce milieu, il l'a été beaucoup plus fortement dans le passé.

De nos jours le Patro dispose d'un réseau dense de petits mouvements locaux, ce qui les distingue nettement des scouts et guides dont les unités sont généralement plus grandes, et donc moins adaptées à un maillage fin du territoire dans un contexte de densités faibles. Notons également que la présence peu marquée de la bourgeoisie dans les espaces ruraux, comme les Ardennes, et un arbitrage de l'église visant à limiter la concurrence entre les mouvements n'a pas favorisé l'implantation F.C.S. ou G.C.B. et a donc laissé le champ relativement libre au développement des patros. Cet arbitrage fut mis en place par les prêtres locaux dès l'apparition des premières unités de scouts et guides catholiques. En fonction de leur appartenance sociale, les jeunes étaient orientés, afin de retrouver une certaine bourgeoisie parmi les scouts et guides catholiques et une jeunesse d'origine populaire au sein des patros. Suite à l'appui du 
pilier catholique via les structures paroissiales, on observe actuellement que seuls les mouvements de jeunesse chrétiens ont pu s'implanter en milieu rural (mis à part les G.C.B.). On retrouve d'ailleurs 3,3\% de jeunes de 5-24 ans des communes rurales fréquentant le Patro et les mêmes chiffres pour la F.C.S.

En dehors d'une spécificité rurale forte, on remarque que la majorité des patros $(33,4 \%$ des effectifs) se trouvent en province de Liège, et plus particulièrement dans les communes opérant une transition entre le milieu urbain et le milieu rural. C'est ainsi qu'on en retrouve un grand nombre à la périphérie de la ville, dans de nombreuses petites paroisses. Alors que les scouts et surtout guides catholiques sont plutôt concentrés, comme nous l'avons déjà précisé, dans les espaces les plus urbanisés.

\section{Scouts et Guides pluralistes et Faucons Rouges : un ancrage territorial très partiel}

\section{Les Faucons Rouges, une expansion spatiale contrôlée par le pilier socialiste}

41 Scouts et Guides Pluralistes sont, comme les Faucons Rouges, nettement moins nombreux que les 3 mouvements précédents. Ils ont tous deux des ancrages sous régionaux marqués.

42 C'est dans le Hainaut que les Faucons Rouges sont les plus représentés proportionnellement à leur effectif total : cette province regroupe en effet près de la moitié de leurs unités contre un quart en moyenne pour les autres mouvements. Ce nombre important de sections locales correspond à une présence historique du mouvement, grâce à l'appui des Mutualités Socialistes qui jusqu'en 1966 (date de la rupture) assuraient le recrutement et l'appui aux structures des Faucons Rouges. Dès son début le mouvement entretenait des liens très étroits avec le Parti Socialiste, notamment par son financement et l'affiliation de ses animateurs au parti. Suite à l'évolution des moeurs, et par volonté d'ouverture, les Faucons Rouges choisirent de prendre leurs distances avec la politique, ce qui se traduira par la création d'un nouveau mouvement de jeunes au sein même des Mutualités Socialistes. Les concentrations secondaires du mouvement se situent dans la partie liégeoise du sillon wallon et dans les communes de l'ouest de Bruxelles.

Les Faucons Rouges sont apparus dans les années 1930 avec pour objectif de diffuser l'esprit socialiste auprès de la jeunesse. Clairement implantés en milieu ouvrier dès l'origine, ils militaient à l'époque pour l'amélioration des conditions de vie des ouvriers et de leur famille (Delporte, date inconnue, p.10). D'une importance numérique comparable à celle des scouts et guides pluralistes, les Faucons Rouges comptabilisaient 4339 membres et 47 sections locales en 2002.

Au même titre que le Patronage et l'Eglise, les Faucons Rouges et le Parti Socialiste ont longtemps entretenu une relation étroite. Ce lien privilégié a facilité l'expansion du mouvement, tout en limitant son public potentiel en raison d'une image teintée de militantisme. Les Faucons Rouges ont été créés sous l'impulsion de membres des Mutualités Socialistes ${ }^{6}$ et encadrés à l'origine par des hauts responsables de ces mêmes mutualités. Jusqu'en 1966, les Faucons Rouges ont fonctionné en étroite relation avec elles, bénéficiant de leurs locaux, subsides ou lieux de recrutement. Il en résulta une 
diffusion spatiale calquée sur l'implantation du pilier socialiste, avec une présence significative dans les zones urbaines ouvrières de l'axe wallon et une très faible pénétration du monde rural ou urbain aisé.

\section{Les scouts et guides pluralistes : une diffusion inachevée}

Actuellement la présence des Scouts et Guides Pluralistes est très faible à Bruxelles et dans le Brabant Wallon par rapport à leurs homologues catholiques. C'est largement dû au fait que le mouvement n'a pour ainsi dire plus grandi après 1975, ceci s'expliquant par un appui devenu inexistant du pilier libéral, ainsi qu'une diversification sans précédent des loisirs proposés à la jeunesse. Toutefois, la capitale rassemble aujourd'hui près de $36 \%$ des effectifs de cette fédération et ils sont bien présents tant dans les quartiers aisés que dans les quartiers plus défavorisés.

Premier mouvement de jeunesse en tant que tel, les scouts et guides pluralistes apparaissent en 1910, avec la première unité en dehors de Grande-Bretagne. Dans un contexte de lutte anticléricale, ils revendiquaient leur neutralité et ne voulaient poursuivre aucun but religieux ou politique.

C'est de nouveau grâce à une analyse des rapports historiques entretenus avec les piliers, que l'on peut expliquer la diffusion spatiale du mouvement. Dès leur création, les scouts et guides pluralistes, qui à l'époque étaient séparés en deux fédérations distinctes, ont pu bénéficier de certains contacts avec le pilier libéral. Dans les années 1920 ces relations avec le pilier libéral se sont concrétisées, en particulier à Bruxelles, par une diffusion accrue dans le réseau des écoles officielles.

On retiendra également que les contacts avec le pilier libéral disparurent assez rapidement. Cette prise de distance mutuelle s'explique par le fait que le mouvement voulait à tout prix rester apolitique et ne désirait pas qu'on lui reproche des connivences avec les libéraux ${ }^{7}$. Le pilier libéral ne voulait pas non plus gérer l'expansion des scouts et guides pluralistes, comme le pilier catholique l'avait fait pour les mouvements de jeunesse chrétiens. De plus, les libéraux ne disposaient pas d'un réseau aussi structuré que le pilier catholique (dont une de ses forces est l'importance du réseau paroissial et des associations gravitant autour), qui aurait pu leur permettre de s'implanter en dehors des grands pôles urbains.

La Fédération des Scouts et Guides Pluralistes (SGP) a été également la seule fédération à avoir établi un réel plan d'expansion spatiale durant les années 1970, plan qui est encore en application aujourd'hui. Cela nous montre bien qu'il y avait au sein du mouvement un sentiment de diffusion inachevée, ce qui n'était pas le cas pour les autres fédérations catholiques, qui à l'époque avaient déjà toutes clôturé leur diffusion spatiale. En 2001, les Scouts et Guides Pluralistes comptaient 3889 membres répartis dans 61 unités.

\section{Synthèse et perspectives}

\section{Une géographie socialement différenciée}

50 L'analyse de la répartition spatiale des mouvements de jeunesse a mis en évidence une ségrégation socio-spatiale largement déterminée par le profil de recrutement des scouts et guides catholiques, majoritaires. Ce clivage social est observé très tôt dans les 
mouvements de jeunesse. Dès les années 1930, les dirigeants des scouts catholiques comprirent qu'il leur serait difficile d'atteindre la population ouvrière à cause des réticences de l'Eglise qui préférait la diriger vers les patronages. Ils se tournèrent donc vers une population plus aisée qui, jusqu'en 1929, n'était pas vraiment concernée par le phénomène des «mouvements de jeunesse $»^{8}$. C'est ainsi que leurs premiers lieux de recrutement furent les collèges d'enseignement catholique. Les scouts catholiques furent donc longtemps implantés dans des milieux plus aisés. Ils n'essayèrent de s'ouvrir à des milieux moins nantis qu'à partir des années 60. Cependant, même si l'on note certaines percées dans ces milieux, notamment dans la région industrielle de Charleroi, la plus forte pénétration structurelle dans les milieux aisés ne s'est pas inversée aujourd'hui. C'est ainsi que l'on atteint encore de nos jours 7,1\% de 5-24 ans fréquentant la F.C.S. dans les communes à revenu fiscal élevé, ce qui contraste nettement avec les taux dans les communes moins aisées.

51 Cette situation de forte dichotomie se vérifie également chez les G.C.B., où on retrouve un rapport de $1 / 5$ entre la fréquentation dans les communes à revenu faible et élevé. L'inertie des localisations et la reproduction sociale pérennisent cette situation. Les coûts de participation aux activités (achat de l'uniforme, cotisation, prix des activités...), souvent cités comme facteur limitant, exercent un effet similaire. Il apparaît en effet que les guides (et les scouts catholiques) sont bien au-dessus du coût moyen demandé par les autres mouvements. Cela joue inévitablement sur la fréquentation de certaines populations, pour qui la contrainte financière est trop importante.

Quant au Patro et aux Faucons Rouges, ce sont les deux seuls mouvements qui ont clairement recruté dans des milieux moins aisés. Leurs meilleurs taux de recrutement s'observent dans des communes au revenu fiscal moyen. En réponse à cette situation, les autres mouvements tentent de diversifier leur public, avec notamment une initiative récente de la F.C.S. et des SGP de promouvoir le scoutisme dans des milieux défavorisés.

La phase de diffusion du scoutisme étant aujourd'hui clairement terminée, tout mouvement désirant élargir sa base de recrutement, tant spatiale que sociologique, ces deux aspects étant évidemment liés, se place automatiquement dans une logique de concurrence avec les autres mouvements.

\section{Un renforcement des concurrences}

Depuis la fin des années 1970, les mouvements de jeunesse, à l'exception de la F.C.S., ont perdu de nombreux membres. Le Patro par exemple a vu ses effectifs se réduire de près de 30000 jeunes en 30 ans. Les chiffres récents montrent une certaine stabilisation, qui reste toutefois précaire en raison d'une offre de loisirs alternatifs de plus en plus large, que ce soit à domicile ou ailleurs.

55 A l'heure actuelle, les efforts des différents mouvements sont essentiellement concentrés sur la solidification des implantations locales, car on remarque depuis 3 ou 4 ans, une baisse nette de fréquentation. Il est peu vraisemblable que dans un avenir proche soient mis en place de nouveaux plans d'expansion spatiale (excepté pour les Scouts et Guides Pluralistes), car il est très difficile de créer de nouvelles implantations sans appuis locaux. Et on observe que là où les mouvements sont absents aujourd'hui, c'est en grande partie en raison du manque de soutien des structures locales. 
56 Enfin, tout au long de cette analyse, nous avons vu que les mouvements de jeunesse évoluent chacun suivant leur propre dynamique et sans réelle concertation (en fait plutôt dans une logique de concurrence), même si des arbitrages ont existé au sein de l'obédience catholique. Compte tenu des aléas qui peuvent fragiliser un mouvement au niveau local (renouvellement des cadres, perte de locaux), on peut s'interroger sur l'opportunité de la poursuite d'une concurrence et de ses effets futurs sur le développement des activités. A terme, des concentrations semblent inévitables, que ce soit au niveau local par disparition et/ou fusion d'unités ou au niveau global par rapprochement des fédérations.

L'introduction de la mixité apparaît également comme un facteur expliquant partiellement la géographie des deux principaux mouvements (F.C.S. et G.C.B.). L'introduction plus tardive de la mixité à la G.C.B. a favorisé dans les espaces les moins densément peuplés une concentration à l'échelle locale en faveur de la FSC. Elle a aussi pénalisé la diffusion des G.C.B. face au F.C.S. dans les espaces de forte périurbanisation du Brabant wallon, pourtant particulièrement favorables sur le plan sociologique.

Aujourd'hui, face à la mixité partagée entre les deux mouvements, les Guides Catholiques de Belgique ont dû se repositionner. La séparation des sexes, qui avait fait leur complémentarité avec leurs homologues masculins, n'a aujourd'hui plus de sens. Sur le plan spatial, la cohabitation au niveau local des deux mouvements génère une concurrence plus forte que jamais.

\section{Les piliers : infrastructure et arbitrage}

Il ressort également de notre analyse que l'implantation des mouvements de jeunesse s'est établie selon des logiques spatiales significativement liées à l'attachement aux piliers. De manière générale, l'expansion spatiale d'un mouvement ne peut se faire à l'échelle de la Belgique francophone sans l'appui d'un de ces piliers. Cela se vérifie pour les cinq fédérations abordées et est particulièrement sensible pour les 3 mouvements d'obédience catholique. Malgré le recul progressif de la pratique "active» du catholicisme et la relative autonomisation de sa sphère associative, l'obédience catholique est encore largement majoritaire dans l'encadrement de jeunes dans les mouvements de jeunesse... tout comme dans l'enseignement d'ailleurs.

Les mouvements catholiques ont suivi un modèle d'expansion très structuré, propre à l'Eglise et largement appuyé sur les paroisses et leur tissu associatif. Chez les Scouts et Guides Pluralistes, on entrevoit assez bien la corrélation entre leur présence initiale sur Bruxelles et leur attachement au pilier libéral. Suite aux distances prises avec celui-ci, on observe que leur expansion a été spatialement limitée. Enfin, les Faucons Rouges se sont nettement implantés avec l'aide du mouvement socialiste et de ses mutualités.

61 A cette logique de support « logistique » s'ajoute, pour le pilier catholique, une logique d'arbitrage. Dans les zones de faible densité, l'Eglise a clairement évité toute concurrence stérile en limitant le développement des Scouts et Guides Catholiques, permettant ainsi au Patro de se maintenir. Cet arbitrage, combiné à la faible présence de la moyenne bourgeoisie en milieu rural, est largement à l'origine de la spécificité rurale du Patro. Le fait de trouver dans certaines régions un seul mouvement de jeunesse a évidemment favorisé une diversification de la population touchée, puisque le choix entre les mouvements ne pouvait se faire. 
62 Le déclin de l'influence directe du pilier catholique au travers des paroisses est néanmoins très net, de manière plus précoce pour les Scouts et Guides Catholiques que pour les Patros. Dans les années 1970, un phénomène généralisé de laïcisation des membres des mouvements de jeunesse catholiques s'observe. Face à cette laïcisation, aussi bien à la base qu'à la tête des mouvements, les fédérations ont adapté leurs contenus pédagogiques et de grands changements ont été mis en oeuvre en matière d'animation spirituelle, qui ne recourt plus systématiquement à un prêtre. Suite à cette scission, beaucoup de mouvements locaux eurent des difficultés à gérer le recrutement de leurs membres. N'ayant plus de personne stable pour superviser le renouvellement des animateurs, certaines implantations périclitèrent. Une grande partie des subsides paroissiaux disparurent également ${ }^{9}$. Ce qu'ils gagnaient en "modernité » d'un côté avec la distanciation avec l'Eglise, les mouvements de jeunesse catholiques le perdaient de l'autre avec la disparition des apports structurels. Ce phénomène a eu ensuite beaucoup d'ampleur au niveau des patros, qui plus que les autres, étaient attachés à la paroisse.

63 Le pilier catholique continue toutefois à structurer les mouvements, mais de manière indirecte notamment via l'enseignement catholique. L'enseignement constitue aussi un canal de recrutement pour les Scouts et Guides Pluralistes, non plus sur une base volontariste comme au $\mathrm{XX}^{\mathrm{e}}$ siècle, mais de manière informelle via le bouche à oreille des enfants.

\section{BIBLIOGRAPHIE}

CHOLVY G. (1988), « Le Patronage : ghetto ou vivier? », Actes du Colloque des 11 et 12 mars 1987, Ed. Nouvelle Cité, 368 p.

COUTROT A. (1985), « Le mouvement de jeunesse, un phénomène au singulier ? », in CHOLVY G. (dir.), Mouvements de jeunesse chrétiens et juifs : sociabilité juvénile dans un cadre européen 1799-1968, Ed. du Cerf, Paris, pp. 109-123.

DELPORTE J. (date inconnue), Les Faucons Rouges. Origines-Evolutions-Philosophie-Structures, 43 p. GEUBELLE P.-L. (1981), in Le P'TIT BIP, Fédération des Eclaireuses et Eclaireurs, Avril, n 25.

JUES J.P. (1996), Le scoutisme, Coll. Que sais-je ?, Paris, P.U.F., 127 p.

LANEYRIE P. (1985), Les scouts de France, l'évolution du mouvement des origines aux années 80, Ed. du Cerf, Paris.

MOREL G. (1987), Notre histoire... ou 75 ans de scoutisme : FSC-SBPB, Bruxelles, 185 p.

MERENNE-SCHOUMAKER B., VAN DER HAEGEN H., VAN ECKHE E. (1998), Monographie 11A : urbanisation, Institut National de Statistique, Bruxelles.

VANDERMOTTEN C. \& KESTELOOT C. (2001), Structures sociales et quartiers en difficulté dans les régions urbaines belges, Politique des grandes villes, $108 \mathrm{p}$. 
VANDERMOTTEN C., DECROLY J.M., DESSOUROUX, C., ROUYET Y. (2001), « Permanences et ruptures dans la géographie électorale de la Belgique », Bruxelles, Belgeo, 1-2, pp. 7-39.

VANDERMOTTEN C. et al. (2005, à paraître), Atlas de Belgique : Géographie politique, Comité National de Géographie et Politique Scientifique Fédérale, Bruxelles.

WINDELS-ROSART F. (1985), « Les mouvements de jeunesse catholiques en Belgique francophone ", in CHOLVY G. (dir.), Mouvements de jeunesse chrétiens et juifs : sociabilité juvénile dans un cadre européen 1799-1968, Ed. du Cerf, Paris, pp. 179-185.

\section{Mémoires}

DUBRUILLE D. (2002), Une géographie des mouvements de jeunesse. Analyse des logiques de répartition spatiale en communauté française de Belgique, mémoire de licence, ULB, 95 p.

FRANS B. (date inconnue), Une jeunesse encadrée : le scoutisme en Belgique (Des origines à la crise des années vingt), mémoire de licence, UCL, $239 \mathrm{p}$.

LEPAGE E. (1986), Histoire de la Fédération Nationale des Patronages. Une réponse du monde catholique au problème populaire, 1922-1940, mémoire de licence UCL, Tomes 1 et 2, $382 \mathrm{p}$.

PREYS D. (1984-1985), Le Patro : mouvement de jeunesse catholique, mémoire de licence, ULB, 100 p.

STAVAUX P. (1994-1995), Le guidisme catholique en Belgique : d'une guerre à l'autre 1915-1939, mémoire de licence, UCL, $133 \mathrm{p}$.

VANDERBECK S. (1983), Evolution de la Fédération des Scouts Catholiques, mémoire de licence, ULB, $130 \mathrm{p}$.

\section{Entretiens}

BROUILLARD Michel, responsable national de la Régionale de Bruxelles 1987-1991, Président national F.N.P.1991-1995 (19/07/02).

DE WAELE Benoît, Secrétaire Fédéral F.C.S.en 2002 (27/06/02).

HEUSCHEN Philippe, Secrétaire Général des Faucons Rouges en 2002 (10/07/02).

RYCKMANS François, cadre fédéral, adjoint au Président F.S.C. 1979-1984, membre de la Commission des Règlements et Formateurs en 2002 (20/07/02).

SCAILLET Thierry, préparant une thèse sur L'histoire de la F.S.C. de Belgique depuis l'entre-deuxguerres jusqu'aux années soixante, archiviste au Centre Historique Belge du Scoutisme (17/07/02). TORCK Maurice, Directeur Général S.G.P. depuis 1991 (18/07/02).

WITTEMANS Sophie, membre de la Commission Histoire, Cheftaine Fédérale G.C.B. 1998-2001 (17/07/02).

\section{NOTES}

1. Scaillet T., interview du 17/07/02.

2. Ryckmans F., interview du 20/07/02.

3. Wittemans S., interview du 16/07/02.

4. Wittemans S., interview du 16/07/02.

5. Scaillet T., interview du 17/07/02.

6. Heuschen P., interview du 10/07/02.

7. Torck M., interview du 18/07/02. 
8. Scaillet T., interview du 17/07/02.

9. Brouillard M., interview du 19/07/02.

\section{RÉSUMÉS}

L'article examine la géographie en Communauté française de Belgique des mouvements de jeunesse relevant du scoutisme, organisations constituées de plusieurs groupements locaux ayant pour objet, l'éducation, l'animation et l'occupation d'enfants ou de jeunes à travers d'un ensemble d'activités variées. Il examine les caractéristiques (urbanité, niveau socio-économique, sous-régionalisme) de l'implantation des différents mouvements et interprète cette répartition comme le résultat d'un processus de diffusion spatiale structuré notamment par les piliers sociopolitiques de la société belge (catholicisme, socialisme, libéralisme).

The paper looks into the geography of scouting movements in the French Community of Belgium, made up of organisations gathering local associations aimed at educating and occupying children or youngsters by means of various activities. We examine the characteristics of the establishment of the different movements (urbanity, socio-economic level, subregionalism) and interpret this distribution as the result of a spatial diffusion process partly structured by the sociopolitical pillars of the Belgian society (catholicism, socialism, liberalism).

\section{INDEX}

Mots-clés : scoutisme, mouvement de jeunesse, encadrement socio-culturel, diffusion spatiale, Communauté française de Belgique

Keywords : scouting, youth movements, socio-cultural activities, spatial diffusion, French Community of Belgium

\section{AUTEURS}

\section{DOMINIQUE DUBRUILLE}

Scouts et Guides Pluralistes, dominique@sgp.be

\section{BENJAMIN WAYENS}

IGEAT, Université Libre de Bruxelles, bwayens@ulb.ac.be

JEAN-MICHEL DECROLY

IGEAT, Université Libre de Bruxelles, jmdecrol@ulb.ac.be 